\title{
Capillary Drops on an Inhomogeneous surface
}

L. A. Caffarelli ${ }^{1 *}$ and A. Mellet ${ }^{2 \dagger}$

1 Dept of Mathematics, University of Texas at Austin, Austin, TX 78712, USA caffarel@math.utexas.edu

2 Dept of Mathematics, University of Texas at Austin, Austin, TX 78712, USA mellet@math.utexas.edu

Summary. We investigate some properties of equilibrium liquid drops lying on a horizontal plane, when small periodic perturbations arise in the properties of that plane (due for example to chemical contamination or roughness). We prove the existence of a minimizer for the energy functional and study its regularity. Then, we show that the free interface stays in a small neighborhood of a portion of sphere (corresponding to the equilibrium drop seating on a homogeneous plane), thus showing the existence of sphere-like capillary drops.

\section{Introduction}

The energy of a drop described by the set $E \subset \mathbb{R}^{n+1}$ and resting on a horizontal plane $(z=0)$ is given by

$$
\sigma \iint_{z>0}\left|D \varphi_{E}\right|-\sigma \int_{z=0} \beta \varphi_{E}(x, 0) d x+\int_{z>0} \rho \Gamma \varphi_{E} d x
$$

where $\varphi_{E}$ is the characteristic function of $E, \sigma$ is the surface tension, $\beta$ is the relative adhesion coefficient between the fluid and the solid, $\Gamma$ is the gravitational energy and $\rho$ is the local density of the fluid. In this paper, we neglect the effect of gravity and assume

$$
\Gamma=0 .
$$

The Euler-Lagrange equation associated to the minimization of (1) under a volume constrain gives rise to a mean-curvature equation, together with a contact angle condition (see [Fin86]). This last condition, known as the Young-Laplace equation reads:

$$
\cos \gamma=\beta,
$$

* L. Caffarelli is partially supported by NSF grant DMS-0140338.

${ }^{\dagger}$ A. Mellet is partially supported by NSF grant DMS-0456647. 
where $\gamma$ denotes the angle between the free surface of the drop $\partial E$ and the horizontal plane $\{z=0\}$ along the contact line $\partial(E \cap\{z=0\})$ (measured within the fluid). The coefficient $\beta$ is determined experimentally and depends on the properties of the materials (solid and liquid). It is usually assumed to be constant, but it is very sensitive to small perturbations in the properties of solid plane (chemical contamination or roughness). These inhomogeneities are responsible for many interesting phenomena such as contact angle hysteresis and sticking drop on inclined surfaces (see [JdG84], [LJ92]). In [HM77], C. Huh and S. G. Mason investigate the effect of roughness of the solid surface on the equilibrium shape of the drop by solving approximately the Young-Laplace equation, for some particular type of periodic roughness.

The purpose of this paper is to investigate the properties of the equilibrium drop in the case of general periodic inhomogeneities, that is when the relative adhesion coefficient satisfies

$$
\beta=\beta(x / \varepsilon),
$$

with $y \mapsto \beta(y) \mathbb{Z}^{n}$-periodic.

The existence of equilibrium drops (minimizing the energy functional) will be shown, within the class of sets of locally finite perimeter. Then, we will investigate the properties of that minimizer, showing in particular that the contact line has finite Hausdorff measure. Finally, we will prove that the equilibrium drop converges uniformly to a spherical cap when the size of the inhomogeneities $(\varepsilon)$ goes to zero (homogenization limit).

Note that this implies the existence of "sphere-like" viscosity solutions to the following free boundary problem (which is the Euler-Lagrange equation for the minimization of (1) when $\partial E$ is a graph):

$$
\begin{gathered}
\operatorname{div}\left(\frac{D u}{\sqrt{1+|D u|^{2}}}\right)=\kappa \quad \text { in }\{u>0\} \\
\frac{D u}{\sqrt{1+|D u|^{2}}} \cdot \nu=\beta(x / \varepsilon) \text { on } \partial\{u>0\} .
\end{gathered}
$$

In $[\mathrm{CM}]$, we investigate further consequences of those results, justifying in particular the two phenomena that we mentioned earlier: Contact angle hysteresis and sticking drop on an inclined plane (for non-vanishing gravity).

\section{Notations and definitions}

\subsection{Sets of finite perimeter}

We recall here the main facts about sets of finite perimeter and BV functions. The standard reference for BV theory is Giusti [Giu84]. Let $\Omega$ be an open subset of $\mathbb{R}^{n+1} ; B V(\Omega)$ denotes the set of all functions in $L^{1}(\Omega)$ with bounded variation: 


$$
B V(\Omega)=\left\{f \in L^{1}(\Omega): \int_{\Omega}|D f|<+\infty\right\} .
$$

where

$$
\int_{\Omega}|D f|=\sup \left\{\int_{\Omega} f(x) \operatorname{div} g(x) d x: g \in\left[C_{0}^{1}(\Omega)\right]^{n+1},|g| \leq 1\right\} .
$$

If $E$ is a Borel set, and $\Omega$ is an open set in $\mathbb{R}^{n+1}$, we recall that the perimeter of $E$ in $\Omega$ is defined by

$$
P(E, \Omega)=\int_{\Omega}\left|D \varphi_{E}\right| .
$$

A Caccioppoli set is a Borel set $E$ that has locally finite perimeter (i.e. $P(E, B)<\infty$ for every bounded open subset $B$ of $\Omega$ ).

Note that sets of finite perimeter are defined only up to sets of measure 0 . We shall henceforth normalize $E$ (as in [Giu84]) so that

$$
0<|\bar{E} \cap B(x, \rho)|<|B(x, \rho)| \quad \text { for all } x \in \partial E \text { and all } \rho>0 .
$$

Furthermore, it is well known that if the boundary $\partial \Omega$ of $\Omega$ is locally Lipschitz, then each function $f \in B V(\Omega)$ has a trace $f^{+}$in $L^{1}(\partial \Omega)$ (see Giusti [Giu84]).

From now on, we denote by $\Omega$ the upper half space:

$$
\Omega=\mathbb{R}^{n} \times(0,+\infty),
$$

and we denote by $(x, z)$ an arbitrary point in $\bar{\Omega}$, with $x \in \mathbb{R}^{n}$ and $z \in[0,+\infty)$.

We denote by $\mathscr{E}(V)$ the class of closed Caccioppoli sets in $\Omega$ with total volume $V>0$ :

$$
\mathscr{E}(V)=\left\{E \subset \Omega: \int_{\Omega}\left|D \varphi_{E}\right|<+\infty,|E|=V\right\},
$$

where $|E|=\int_{\Omega} \varphi_{E}$ demotes the Lebesgue measure of $E$. Since Caccioppoli sets have a trace on $\partial \Omega=\mathbb{R}^{n} \times\{z=0\}$, we can define the following functional for every $E \in \mathscr{E}(V)$ :

$$
\begin{aligned}
\mathcal{J}(E) & =\iint_{z>0}\left|D \varphi_{E}\right|-\int_{z=0} \beta(x) \varphi_{E}(x, 0) d x \\
& =P(E, \Omega)-\int_{E \cap\{z=0\}} \beta(x) d \mathscr{H}^{n}(x) .
\end{aligned}
$$

In this framework, equilibrium liquid drops are solutions of the minimization problem:

$$
\mathcal{J}(E)=\inf _{F \in \mathscr{E}(V)} \mathcal{J}(F)
$$




\subsection{Constant adhesion coefficient}

When $\beta=\beta_{o}$ is constant, the existence of a minimizer was established by $\mathrm{E}$. Gonzalez [Gon76]. The corresponding functional reads

$$
\mathcal{J}_{o}(E)=\int_{\Omega}\left|D_{\varphi_{E}}\right|-\beta_{o} \int \varphi_{E}(x, 0) d x .
$$

When $\beta_{o}=-1$ (hydrophobic surface), the absolute minimizers are the spheres of volume $V$ in $\{z>0\}$. In particular, the equilibrium drop does not touch the support plane. On the contrary, if $\beta_{o}>-1$, it is easy to see that, though a sphere of volume $V$ is still a local (degenerate) minimizer of the functional $\mathcal{J}$, the absolute minimizer must touch the solid support.

An important tool, when the adhesion coefficient is constant, is the Schwartz symmetrization (see [Gon76]): For every $E \in \mathscr{E}(V)$, the set

$$
E^{s}=\{(x, z) \in \Omega ;|x|<\rho(z)\}, \quad \text { where } \rho(z)=\left(\omega_{n}^{-1} \int \varphi_{E}(x, z) d x\right)^{\frac{1}{n}}
$$

is a Caccioppoli set with volume $V$ satisfying

$$
\mathcal{J}_{o}\left(E^{s}\right) \leq \mathcal{J}_{o}(E)
$$

with equality if and only if $E$ was already symmetric. This clearly implies that any minimizer should have axial symmetry. Actually, it can be shown that the minimizers are spherical caps; that is the intersection of a ball $B_{\rho_{o}}\left(0, z_{o}\right)$ in $\mathbb{R}^{n+1}$ with the upper-half space $\Omega$. We denote by

$$
B_{\rho_{o}}^{+}\left(z_{o}\right)=B_{\rho_{o}}\left(0, z_{o}\right) \cap\{z>0\}
$$

such a spherical cap.

Our main result is a stability/uniqueness result for the minimization problem with constant coefficient:

Theorem 1. Let $E$ be such that

$$
\begin{aligned}
& E \in \mathscr{E}(V), \quad E \text { lies in a bounded subset } B_{R} \text { of } \Omega, \\
& \exists \delta>0 \quad \text { s.t. } \mathcal{J}_{o}(E) \leq \mathcal{J}_{o}(F)+\delta \quad \forall F \in \mathscr{E}(V) .
\end{aligned}
$$

Then there exists a universal $\alpha>0$ and a constant $C$ (depending on $R$ ) such that

$$
\left|E \Delta B_{\rho_{o}}^{+}\right| \leq C \delta^{\alpha}
$$

where $B_{\rho_{o}}^{+}$is such that $\left|B_{\rho_{o}}^{+}\right|=V$ and the cosine of the contact angle is $\beta_{o}$.

Note that we recover in particular the fact that the gravity-free equilibrium drop is a constant mean-curvature surface satisfying the Young-Laplace condition. 
If moreover $E$ satisfies some non-degeneracy conditions, then Theorem 1 implies the uniform stability in the following sense: For any $\eta>0$, there exists $\delta_{o}$ such that if $(7)$ holds with $\delta<\delta_{o}$, then

$$
B_{(1-\eta) \rho}^{+} \subset E \subset B_{(1+\eta) \rho}^{+} .
$$

In other words, the free surface $\partial E \cap\{z>0\}$ stays between $\partial B_{(1+\eta) \rho}^{+}$and $\partial B_{(1-\eta) \rho}^{+}$.

\subsection{Periodic adhesion coefficient}

First of all, note that when the relative adhesion coefficient $\beta$ depends on $x$, the rearrangement (5) could increase the wetting energy

$$
\int \beta(x) \varphi_{E}(x, 0) d x
$$

However, if $\beta=\beta(x / \varepsilon)$ is periodic with small period $\varepsilon$, the wetting energy should not increase by more that $C \varepsilon$ after symmetrization. If we can prove that fact, Theorem 1 will allow us to show that the minimizer associated with a periodic adhesion coefficient is almost a spherical cap (in $L^{1}$ and $L^{\infty}$ ).

We now make our framework precise: We consider the following energy functional:

$$
\mathcal{J}(E)=\iint_{z>0}\left|D \varphi_{E}\right|-\int_{z=0} \beta(x / \varepsilon) \varphi_{E}(x, 0) d x .
$$

where $\beta$ satisfies

$$
-1<\beta(x)<1, \quad \text { for all } x \in \mathbb{R}^{n}
$$

and

$$
x \mapsto \beta(x) \text { periodic }
$$

We denote by $\langle\beta\rangle$ the average of $\beta$ :

$$
\langle\beta\rangle=\int_{[0,1]^{n}} \beta(x) d x,
$$

and by $B_{\rho_{o}}^{+}\left(z_{o}\right)=B_{\rho_{o}}\left(0, z_{o}\right) \cap\{z>0\}$ the minimizer of the constant adhesion coefficient functional

$$
\mathcal{J}_{o}(E)=\iint_{z>0}\left|D \varphi_{E}\right|-\int_{z=0}\langle\beta\rangle \varphi_{E}(x, 0) d x .
$$

Then we prove:

\section{Theorem 2.}


(i) For all $V$ and $\varepsilon>0$, there exists $E$ minimizer of $\mathcal{J}$ in $\mathscr{E}(V)$. Moreover, up to a translation, we can always assume that

$$
E \subset\left\{|x| \leq R_{o} V^{\frac{1}{n+1}}, z \in\left[0, T_{o} V^{\frac{1}{n+1}}\right]\right\}
$$

with $R_{o}$ and $T_{o}$ universal constants.

(ii) The contact line $\partial(E \cap\{z=0\})$ has finite $(n-1)$ Hausdorff measure in $\mathbb{R}^{n}$.

(iii) There exists a constant $C$ such that if $\varepsilon \leq C(V) \eta^{(n+1) / \alpha}$, then

$$
B_{(1-\eta) \rho_{o}}^{+} \subset E \subset B_{(1+\eta) \rho_{o}}^{+} .
$$

In other words, the free surface $\partial E \cap\{z>0\}$ lies between $\partial B_{(1+\eta) \rho_{o}}^{+}$and $\partial B_{(1-\eta) \rho_{o}}^{+}$for $\varepsilon$ small enough.

The proof of (i) is very classical and will be developed in Sections 3 and 4; It relies on the properties of $B V$ functions and some monotonicity formulas for the minimizers of $\mathcal{J}$. The regularity result (ii) is established in Section 5 and allows us to show that the energy of the minimizer only differs by $\varepsilon$ from the energy corresponding to the wetting coefficient $\langle\beta\rangle$. The last point will then follow using Theorem 1, the proof of which is presented in Section 6.

\section{Constrained minimizer}

The lower semi-continuity of the functional (2) in the $L^{1}(\Omega)$ topology is easy to establish. Moreover, it is a classical result that bounded sets of $B V$ are pre-compact in $L_{l o c}^{1}(\Omega)$. Those two facts give the convergence of a minimizing sequence to a minimizer if $|\Omega|<+\infty$. Since we are considering drops lying in the upper half space, we can only prove the existence of minimizers if we first restrict ourself to Caccioppoli sets lying in a bounded subset of $\Omega$ (constrained minimizers); this is the object of this section. In Section 4, we will show that this minimizer is actually an unconstrained minimizer

To guarantee the compactness of minimizing sequences, we first look for minimizers that stay within a bounded subset of $\mathbb{R}^{n+1}$. Let

$$
\Gamma_{R, T}=\left\{(x, z) \in \mathbb{R}^{n+1} ;|x| \leq R, z \in[0, T]\right\},
$$

and

$$
\mathscr{E}_{R, T}(V)=\left\{E \in \mathscr{E}(V) ; E \subset \Gamma_{R, T}\right\} .
$$

We call constrained minimizer a set $E \in \mathscr{E}_{R, T}(V)$ satisfying

$$
\mathcal{J}(E)=\min _{F \in \mathscr{E}_{R, T}(V)} \mathcal{J}(F) .
$$

We have the following proposition: 
Proposition 1. If $R$ and $T$ are such that there exists a ball $\widetilde{B}$ with volume $V$ in $\Gamma_{R, T}$, then there exists a minimizer $E_{R, T}$ for (9). Moreover, we have

$$
P\left(E_{R, T}\right) \leq C V^{\frac{n}{n+1}} \quad \text { and } \mathscr{H}^{n}\left(E_{R, T} \cap\{z=0\}\right) \leq C V^{\frac{n}{n+1}} .
$$

The proof is very classical. The key is to establish the lower continuity of the functional in a topology that makes minimizing sequences pre-compact. We recall the following classical result for functions with bounded variation (see [Giu84]):

Lemma 1. Let $\Omega \subset \mathbb{R}^{n}$ be an open set, and $\left(f_{j}\right)$ a sequence of functions in $B V(\Omega)$ which converges in $L_{\text {loc }}^{1}(\Omega)$ to a function $f$. Then

$$
\int_{\Omega}|D f| \leq \liminf _{j \rightarrow \infty} \int\left|D f_{j}\right|
$$

It follows:

Lemma 2. The functional $\mathcal{J}$ is lower continuous with respect to the $L^{1}$ topology: If $\left(E_{j}\right)$ is a sequence of Caccioppoli sets such that $E_{j} \longrightarrow E$ in $L^{1}$ then

$$
\mathcal{J}(E) \leq \liminf _{j \rightarrow \infty} \mathcal{J}\left(E_{j}\right)
$$

Proof: Assume on the contrary that there exists $\delta>0$ such that

$$
\mathcal{J}\left(E_{j}\right) \leq \mathcal{J}(E)-\delta
$$

or

$$
\iint_{z>0}\left|D \varphi_{E_{j}}\right| \leq \iint_{z>0}\left|D \varphi_{E}\right|+\int_{z=0} \beta\left(\varphi_{E}-\varphi_{E_{j}}\right) d x-\delta
$$

We recall the following estimate (see [Giu84]):

$$
\begin{aligned}
\int_{z=0}\left|\varphi_{E}-\varphi_{E_{j}}\right| d x \leq \iint_{\Gamma_{R, t}}\left|D \varphi_{E}\right|+\iint_{\Gamma_{R, t}}\left|D \varphi_{E_{j}}\right| \\
+C(t) \iint_{z>0}\left|\varphi_{E}-\varphi_{E_{j}}\right| d x d z-\delta .
\end{aligned}
$$

The last term clearly goes to zero as $j$ goes to infinity, so

$$
\liminf \iint_{\Omega \backslash \Gamma_{R, t}}\left|D \varphi_{E_{j}}\right| \leq \iint_{z>0}\left|D \varphi_{E}\right|+\iint_{\Gamma_{R, t}}\left|D \varphi_{E}\right|-\delta,
$$

from which we derive a contradiction, taking into account the lower continuity of the perimeter (Lemma 1), and letting $t$ goes to zero.

Next, we recall the following compactness result for functions of bounded variations:

Lemma 3. Let $\Omega$ be a bounded open set in $\mathbb{R}^{n}$ with Lipschitz boundary. Then sets of functions uniformly bounded in $B V$-norm are relatively compact in $L^{1}(\Omega)$. 
The existence of a constrained minimizer is therefore a consequence of the following lemma:

Lemma 4 (A priori estimates). If $-1<\beta<1$, then

$$
\mathcal{J}(E) \geq \frac{1-\beta_{\max }}{2} \int_{z>0}\left|D \varphi_{E}\right|+\frac{1-\beta_{\max }}{2} \int_{z=0} \varphi_{E} d x
$$

for all $E \in \mathscr{E}(V)$.

Note that Lemma 4 and the fact that $\widetilde{B}$ lies in $\Gamma_{R, T}$ give $(10)$, since

$$
\mathcal{J}(E) \leq \mathcal{J}(\widetilde{B})=\mu V^{\frac{n}{n+1}}
$$

Proof: Note that if $g(x)$ is a non-negative function, then

$$
\int_{z>0} g(x)\left|D \varphi_{E}\right| \geq \int_{z=0} g(x) \varphi_{E} d x
$$

Hence

$$
\int_{z>0} \frac{1+\beta(x)}{2}\left|D \varphi_{E}\right| \geq \int_{z=0} \frac{1+\beta(x)}{2} \varphi_{E} d x
$$

and therefore

$$
\int_{z>0}\left(1-\frac{1-\beta(x)}{2}\right)\left|D \varphi_{E}\right| \geq \int_{z=0}\left(\frac{1-\beta(x)}{2}+\beta(x)\right) \varphi_{E} d x
$$

or

$$
\mathcal{J}(E) \geq \int_{z>0} \frac{1-\beta(x)}{2}\left|D \varphi_{E}\right|+\int_{z=0} \frac{1-\beta(x)}{2} \varphi_{E} d x
$$

Lemma 2, 3 and 4, together with the fact that $\mathscr{E}_{R, T}(V) \neq \emptyset$ gives Proposition 1.

We now have to prove that if $R$ and $T$ are large enough, $E_{R, T}$ is in fact an unconstrained minimizer.

\section{Unconstrained minimizer}

In this section we prove the following result:

Proposition 2. There exists $T_{o}$ and $R_{o}$ such that if

$$
R \geq R_{o} V^{\frac{1}{n+1}} \quad \text { and } \quad T \geq T_{o} V^{\frac{1}{n+1}},
$$

then $E_{R, T} \in \mathscr{E}_{R_{o}, T_{o}}$. In particular, $E_{R, T}$ is an unconstrained minimizer.

After rescaling, we only have to prove the result when $|E|=V=1$. 


\subsection{Bounded above.}

First, we prove that for $T$ large enough, the minimizer for $\mathcal{J}$ in $\mathscr{E}_{R, T}(V)$ is actually a minimizer in $\mathscr{E}_{R}(V)$. This follows from the following result:

Proposition 3. There exists $T_{1}$ such that for all $T \geq T_{1}$, there exists a minimizer $E$ of $\mathcal{J}_{R, T}$ with $E \in \mathscr{E}_{R, T_{1}}(V)$.

The proof relies on a slight modification of an argument first presented by E. Barozzi in [Bar83] (see also E. Barozzi and E. Gonzalez, [BG84]). The key lemma is the following:

Lemma 5. There exists $T_{1} \geq T_{o}$ such that for $T \geq T_{1}$ and $E$ minimizer of $\mathcal{J}$ in $\mathscr{E}_{R, T}(V)$, there exists $t, T_{o} \leq t \leq T_{1}$ with

$$
\mathscr{H}^{n}(E \cap\{z=t\})=0 .
$$

Let us recall here that the coarea formula gives

$$
|E|=\int_{0}^{+\infty} \mathscr{H}^{n}(E \cap\{z=t\}) d t,
$$

which in particular implies that

$$
\mathscr{H}^{n}(E \cap\{z=t\})<\infty \quad \text { a.e. } t \in \mathbb{R} .
$$

Proof of Proposition 3: Let $E$ be a minimizer of $\mathcal{J}$ in $\mathscr{E}_{R, T}(V)$, and let $t$ be as in Lemma 5. Then, the part of $E$ that lies above $z=t$ contribute to the perimeter of $E$ by at least

$$
\frac{1}{\mu_{n+1}}|E \cap\{z>t\}|^{\frac{n}{n+1}},
$$

where $\mu_{n+1}$ is the isoperimetric constant. Thus, if we define

$$
E_{o}=\left\{\begin{array}{ll}
E & \text { in }\{0<z<t\} \backslash \widetilde{B} \\
\emptyset & \text { in }\{z>t\} \\
E \cup \rho \widetilde{B} & \text { in } \widetilde{B}
\end{array},\right.
$$

with $\rho$ such that $\left|E_{o}\right|=|E|$, we have

$$
\mathcal{J}\left(E_{o}\right) \leq \mathcal{J}(E)=\min _{F \in \mathscr{E}_{R, T}(V)} \mathcal{J}(F),
$$

and $E_{o} \in \mathscr{E}_{R, T_{1}}(V)$.

Taking $T \rightarrow \infty$, the compactness property of the minimizing sequence gives the existence of a set $E \in \mathscr{E}_{R, T_{1}}(V)$ such that

$$
\mathcal{J}(E)=\min _{F \in \mathscr{E}_{R}(V)} \mathcal{J}(F)
$$


In other words, $E$ is a vertically-unconstrained minimizer.

Proof of Lemma 5: Let $t_{1}, t_{2}$ and $t_{3}$ be three positive numbers with

$$
0<t_{1}<t_{2}<t_{3}<T \text {. }
$$

We denote

$$
\begin{aligned}
& v_{1}=\left|E \cap\left\{t_{1}<z<t_{2}\right\}\right| \\
& v_{2}=\left|E \cap\left\{t_{2}<z<t_{3}\right\}\right|
\end{aligned}
$$

and

$$
m=\max _{i=1,2,3} \mathscr{H}^{n}\left(E \cap\left\{z=t_{i}\right\}\right) .
$$

The isoperimetric inequality yields:

$$
\begin{aligned}
& v_{1}^{\frac{n}{n+1}} \leq \mu\left(2 m+S_{1}\right) \\
& v_{2}^{\frac{n}{n+1}} \leq \mu\left(2 m+S_{2}\right),
\end{aligned}
$$

where $S_{i}$ is the surface of the lateral boundary:

$$
S_{i}=\int_{\left\{t_{i}<z<t_{i+1}\right\}}\left|D \varphi_{E}\right|=P\left(E,\left\{t_{i}<z<t_{i+1}\right\}\right)
$$

In particular, we have:

$$
v_{1}^{\frac{n}{n+1}}+v_{2}^{\frac{n}{n+1}} \leq \mu(4 m+S)
$$

Let us now define the set $F$ by

$$
F=\left\{\begin{array}{ll}
E & \text { in } \Gamma_{R, T} \backslash\left\{t_{1}<z<t_{3}\right\} \backslash \widetilde{B} \\
\emptyset & \text { in }\left\{t_{1}<z<t_{3}\right\} \\
E \cup \rho \widetilde{B} & \text { in } \widetilde{B}
\end{array},\right.
$$

where we recall that $\widetilde{B}$ is a ball with volume $V$ lying in $\Gamma_{R, T}$, and $\rho$ is such that $|F|=|E|$. Then,

$$
\mathcal{J}(F) \leq \mathcal{J}(E)-S+2 m+\frac{1}{\mu}\left(v_{1}+v_{2}\right)^{\frac{n}{n+1}} .
$$

Since $E$ is a minimizer, we deduce:

$$
S \leq 2 m+\frac{1}{\mu}\left(v_{1}+v_{2}\right)^{\frac{n}{n+1}}
$$

Thus, inequalities (11) and (12) implies

$$
v_{1}^{\frac{n}{n+1}}+v_{2}^{\frac{n}{n+1}}-\left(v_{1}+v_{2}\right)^{\frac{n}{n+1}} \leq C m
$$


and therefore

$$
\min \left(v_{1}, v_{2}\right) \leq C m^{\frac{n}{n+1}}
$$

Let $a_{o}=T_{o}$ and $b_{o}=T_{1}$. Given $a_{k}$ and $b_{k}$, we define

$$
v_{k}=\left|E \cap\left\{a_{k}<z<b_{k}\right\}\right| .
$$

Then, for $h_{k}=\frac{b_{k}-a_{k}}{4}$ it is possible to find $t_{1} \in\left(a_{k}, a_{k}+h_{k}\right), t_{2} \in\left(\frac{a_{k}+b_{k}}{2}-\right.$ $\left.\frac{h_{k}}{2}, \frac{a_{k}+b_{k}}{2}+\frac{h_{k}}{2}\right)$ and $t_{3} \in\left(b_{k}-h_{k}, b_{k}\right)$ such that

$$
\mathscr{H}^{n}\left(E \cap\left\{z=t_{i}\right\}\right) \leq \frac{v_{k}}{h_{k}} \quad \text { for } i=1,2,3
$$

Setting

$$
\left\{\begin{array}{l}
v_{i}^{k}=\left|E \cap\left\{t_{i}<z<t_{i+1}\right\}\right|, \quad i=1,2 \\
v_{k+1}=\min \left(v_{1}^{k}, v_{2}^{k}\right)
\end{array}\right.
$$

we choose

$$
\left(a_{k+1}, b_{k+1}\right)=\left\{\begin{array}{l}
\left(t_{1}, t_{2}\right) \text { if } v_{1}^{k} \leq v_{2}^{k} \\
\left(t_{2}, t_{3}\right) \text { if } v_{2}^{k}<v_{1}^{k}
\end{array}\right.
$$

Let

$$
m_{k}=\max _{i=1,2,3} \mathscr{H}^{n}\left(E \cap\left\{z=t_{i}\right\}\right),
$$

It follows from (13) that

$$
\begin{aligned}
& v_{k+1} \leq C m_{k}^{\frac{n+1}{n}} \\
& m_{k} \leq \frac{v_{k}}{h_{k}}
\end{aligned}
$$

and $b_{k+1}-a_{k+1} \geq \frac{b_{k}-a_{k}}{8}$ (and thus $h_{k+1} \geq \frac{h_{k}}{8}$ ). It follows that

$$
m_{k+1} \leq \frac{C}{h_{k}} m_{k}^{\frac{n+1}{n}}
$$

and therefore, with the motation $\alpha=\frac{n}{n+1}$,

$$
\begin{aligned}
m_{k} & \leq \frac{C^{1+\alpha+\alpha^{2}+\cdots+\alpha^{k}}}{h_{k+1} h_{k}^{\alpha} \cdots h_{1}^{\alpha^{k}}} v_{o}^{\alpha^{k+1}} \\
& \leq\left(\frac{C^{\alpha^{-k}+\cdots+1}}{h_{k+1}^{\alpha^{-k}} h_{k}^{\alpha^{-k+1}} \cdots h_{1}} v_{o}\right)^{\alpha^{k}} .
\end{aligned}
$$

Since $h_{k} \geq \frac{b_{o}-a_{o}}{8^{k}}$, we deduce

$$
m_{k} \leq\left(C \frac{v_{o}}{b_{o}-a_{o}}\right)^{\alpha^{k}}
$$

Thus, choosing $T_{1}$ large enough so that 


$$
C \frac{v_{o}}{b_{o}-a_{o}}=C \frac{v_{o}}{T_{1}-T_{o}}<1
$$

we have

$$
m_{k} \longrightarrow 0 \text { as } k \rightarrow \infty,
$$

which conclude the proof of Lemma 5.

\subsection{Monotonicity Formula}

The proof of Proposition 2 now relies on some monotonicity formula that we derive in this section. We start with a simple observation:

$$
\min _{\mathscr{E}_{R}\left(V_{o}\right)} \mathcal{J} \leq \min _{\mathscr{E}_{R}\left(V_{o}+\delta V\right)} \mathcal{J} \leq \min _{\mathscr{E}_{R}\left(V_{o}\right)} \mathcal{J}+V_{o}^{-\frac{1}{n+1}} \delta V .
$$

To establish this fact, take $E$ minimizer of $\mathcal{J}$ in $\mathscr{E}_{R}\left(V_{o}\right)$. The vertical dilatation of $E$ :

$$
\widetilde{E}_{t}=\left\{(x, z) \in \mathbb{R}^{n+1} ;\left(x,(1+t)^{-1} z\right) \in E\right\},
$$

satisfies

$$
\left|\widetilde{E}_{t}\right|=(1+t)|E|, \quad \text { and } \quad P\left(\widetilde{E}_{t}, \Omega\right) \leq(1+t) P(E, \Omega),
$$

but the wetting energy is left unchanged. Thus

$$
\mathcal{J}\left(\widetilde{E}_{t}\right) \leq \mathcal{J}(E)+t P(E, \Omega) .
$$

So if we take $t=\delta V / V_{o}$, we get

$$
\widetilde{E}_{t} \in \mathscr{E}_{R}\left(V_{o}+\delta V\right),
$$

and

$$
\begin{aligned}
\mathcal{J}\left(\widetilde{E}_{t}\right) & \leq \mathcal{J}(E)+t P(E, \Omega) \\
& \leq \min _{\mathscr{E}_{R}\left(V_{o}\right)} \mathcal{J}+C\left(V_{o}\right) \delta V,
\end{aligned}
$$

where $C\left(V_{o}\right)=\frac{1}{V_{o}} P(E, \Omega)$, thus giving the second inequality in (14) (using the fact that $\left.P(E, \Omega) \leq\left|V_{o}\right|^{\frac{n}{n+1}}\right)$.

Similarly, if $F$ is a minimizer of $\mathcal{J}$ in $\mathscr{E}_{R}(V+\delta V)$, then the set

$$
\widetilde{F}_{t}=\left\{(y, z) \in \mathbb{R}^{n+1} ;(y,(1+t) z) \in E\right\},
$$

with $t=\delta V / V$ satisfies

$$
\left|\widetilde{F}_{t}\right|=(1+t)^{-1}|F|=V, \quad \text { and } P\left(\widetilde{F}_{t}, \Omega\right) \leq P(F, \Omega)
$$

and therefore

$$
\mathcal{J}\left(\widetilde{F}_{t}\right) \leq \mathcal{J}(F),
$$


proving (14).

The first monotonicity formula guarantee the non-degenerescence of $E$ near a point of the contact line. It will allow us to show that $E$ lies in a bounded set of $\Omega$. Let $\Gamma_{r}\left(x_{o}\right)$ denote the cylinder

$$
\Gamma_{r}\left(x_{o}\right)=B_{r}^{n}\left(x_{o}\right) \times \mathbb{R}=\left\{(x, z) \in \mathbb{R}^{n+1} ;\left|x-x_{o}\right|<r\right\},
$$

then we have the following:

Lemma 6. There exists $c_{1}, c_{o}>0$ such that for any minimizer $E$ of $\mathcal{J}$ in $\mathscr{E}_{R}(V)$, if $x_{o}$ lies in the projection of $E$ onto $\{z=0\}$ (i.e. there exists $z_{o}$ such that $\left(x_{o}, z_{o}\right) \in E$ ) then

$$
\left|E \cap \Gamma_{r}\left(x_{o}\right)\right|>c_{o} r^{n+1},
$$

for all $r$ such that $\left|E \cap \Gamma_{r}\left(x_{o}\right)\right| \leq c_{1}|E|$.

Proof: We denote

$$
\begin{aligned}
& U(r)=\left|E \cap \Gamma_{r}\left(x_{o}\right)\right|, \\
& S(r)=\mathscr{H}^{n}\left(E \cap \partial \Gamma_{r}\left(x_{o}\right) \cap\{z>0\}\right)
\end{aligned}
$$

Note that $U^{\prime}(r)=S(r)$. We also introduce

$$
A_{1}(r)=P\left(E, \Gamma_{r} \cap\{z>0\}\right)
$$

the area of the free surface in $\Gamma_{r}$ and

$$
A_{2}(r)=\int_{B_{r}^{n}\left(x_{o}\right)} \varphi_{E}(x, 0) d x
$$

the wetted area in $\Gamma_{r}$. Since $E$ is bounded above, if $x \in E \cap\{z=0\}$, going from the slice $\{z=0\}$ to the slice $\{z=T\}$, we must cross $\partial E$. Therefore we have

$$
A_{2} \leq A_{1}
$$

The isoperimetric inequality then gives:

$$
V(r)^{n /(n+1)} \leq \mu\left(2 A_{1}+S(r)\right) .
$$

Consider now the set $F=E \backslash \Gamma_{r}\left(x_{o}\right)$. It satisfies:

$$
\mathcal{J}(F) \leq \mathcal{J}(E)-A_{1}+\beta_{\max } A_{2}+S, \quad \text { and }|F|=|E|-U(r),
$$

and using (14), with $|F|=V_{o}$ and $\delta V=U$, we get

$$
\mathcal{J}(E)=\min _{\mathscr{E}(|E|)} \mathcal{J}=\min _{\mathscr{E}\left(V_{o}+U\right)} \mathcal{J} \leq \mathcal{J}(F)+C(|E|) U(r) .
$$

It follows that 


$$
\min \left(1,1-\beta_{\max }\right) A_{1} \leq A_{1}-\beta_{\max } A_{2} \leq S+C(|F|) U(r),
$$

with $C(F) \leq \frac{1}{V_{o}} P(E, \Omega) \leq C(|E|)$ as long as $U \leq|E| / 2$. Thus

$$
U(r)^{n /(n+1)} \leq C U^{\prime}(r)+C U(r),
$$

and if $U \leq C^{-(n+1)} / 2$, we deduce

$$
U(r)^{n /(n+1)} \leq C U^{\prime}(r),
$$

and Gronwall's Lemma gives the result.

Using similar arguments, we can also establish the following monotonicity formula:

Lemma 7. Let $\left(x_{o}, z_{o}\right) \in \partial E$ with $z_{o}>0$. There exists $c$, universal constant, such that for all $r \leq z_{o}$ we have

$$
\begin{aligned}
& \left|B_{r}\left(x_{o}, z_{o}\right) \cap E\right| \geq c r^{n+1} \\
& \left|B_{r}\left(x_{o}, z_{o}\right) \backslash E\right| \geq c r^{n+1}
\end{aligned}
$$

Proof. For $r \leq z_{o}$, we define

$$
\begin{array}{ll}
U_{1}(r)=\left|B_{r}\left(x_{o}, z_{o}\right) \cap E\right| & S_{1}(r)=\mathscr{H}^{n}\left(\partial B_{r}\left(x_{o}, z_{o}\right) \cap E\right) \\
U_{2}(r)=\left|B_{r}\left(x_{o}, z_{o}\right) \backslash E\right| & S_{2}(r)=\mathscr{H}^{n}\left(\partial B_{r}\left(x_{o}, z_{o}\right) \backslash E\right)
\end{array}
$$

As in the previous proof, the minimality of $E$ and the fact that $B_{r}$ lies entirely in $\Omega$ for $r<z_{o}$ give (by estimating $\mathcal{J}\left(E \backslash B_{r}\right)$ and $\mathcal{J}\left(E \cup B_{r}\right)$ respectively):

$$
\begin{aligned}
& P\left(E, B_{r}\left(x_{o}, z_{o}\right)\right) \leq S_{1}(r)+C U_{1}(r) \\
& P\left(E, B_{r}\left(x_{o}, z_{o}\right)\right) \leq S_{2}(r),
\end{aligned}
$$

which together with the isoperimetric formula yields

$$
\begin{aligned}
& U_{1}(r)^{\frac{n}{n+1}} \leq 2 \mu\left(S_{1}(r)+C U_{1}(r)\right) \\
& U_{2}(r)^{\frac{n}{n+1}} \leq 2 \mu S_{2}(r),
\end{aligned}
$$

Since $U_{i}^{\prime}(r)=S_{i}(r)$, Gronwall's Lemma gives the result.

\subsection{Unconstrained minimizers}

We now complete the proof of Proposition 2: Let $G$ denote the projection of $E$ onto $\{z=0\}$. As a consequence of Lemma 6 , there exists $\rho_{o}=C|E|^{\frac{1}{n+1}}$ such that if $x \in G$, then

$$
\left|E \cap \Gamma_{\rho_{o}}(x)\right| \geq C \rho_{o}^{n+1}
$$


Consider the familly $\left\{B_{\rho_{o}}(x) \mid x \in G\right\}$. We can extract a subfamilly $B_{\rho_{o}}\left(x_{j}\right)$ with finite overlapping still covering $G$. In particular,

$$
\begin{aligned}
|E| & =\left|\bigcup_{j} E \cap \Gamma_{\rho_{o}}\left(x_{j}\right)\right| \\
& \geq C(n) \sum_{j}\left|E \cap \Gamma_{\rho_{o}}\left(x_{j}\right)\right| \\
& \geq C(n) \sum_{j} \rho_{o}^{n+1} .
\end{aligned}
$$

This implies that the subfamilly contains at most $|E| / \rho_{o}{ }^{n+1}$ balls. Using the periodicity of $\beta$, we deduce that $G$ has at most radius

$$
\frac{|E|}{C \rho_{o}^{n+1}} \rho_{o}=C|E|^{\frac{1}{n+1}} .
$$

So the proof of Proposition 2 is complete.

\section{Properties of the minimizers}

In this section, we investigate the regularity of the minimizer $E$. The regularity of the free surface $\partial E \cap \Omega$ is a consequence of classical regularity results for minimal surface. We then determine the Hausdorff dimension of the contact line $\partial E \cap\{z=0\}$.

\subsection{Regularity of the free surface.}

Note that if $E$ is a solution of (3), then $E$ also minimizes the functional

$$
\mathcal{F}(F)=\int_{\Omega}\left|D \varphi_{F}\right|+\int_{z=0}\left|\varphi_{F}-\varphi_{E}\right| d x
$$

among the Caccioppoli subsets of $\Omega$ satisfying $|F|=|E|$. We can therefore apply the classical regularity results for minimal surfaces (see E. Gonzalez et al. [GMT83]):

Theorem 3. If $E$ minimizes the functional $\mathcal{J}$ in $\mathscr{E}(V)$, then $\partial^{*} E \cap \Omega$ is an analytic (n-1)-manifold and $H^{s}\left[\left(\partial E \backslash \partial^{*} E\right) \cap \Omega\right]=0$ for all $s>n-8$. In particular, if $n+1 \leq 7$, the singular set is empty.

\subsection{Hausdorff measure of the contact line}

We now establish the following proposition: 
Proposition 4. The contact line $\partial(E \cap\{z=0\})$ in $\mathbb{R}^{n}$ has finite $n-1$ Hausdorff measure in $\mathbb{R}^{n}$ and

$$
\mathscr{H}^{n-1}(\partial(E \cap\{z=0\})) \leq C V^{\frac{n-1}{n+1}} .
$$

The proof relies on the monotonicity formula and a couple of lemma. We start with the following:

Lemma 8. Let $x_{o}$ be a point in $\mathbb{R}^{n}$. There exists a critical $\delta_{o}>0$ such that if

$$
B_{r}^{+}\left(x_{o}, 0\right) \backslash\left\{z<\delta_{o} r\right\} \subset E,
$$

then

$$
B_{r / 2}^{+}\left(x_{o}, 0\right) \subset E
$$

Proof. Let us renormalize and take $r=1$. The proof relies on a De Giorgi type argument: Consider the vertical cylinders

$$
\Gamma_{k}=B_{r_{k}}^{n} \times \mathbb{R}, \quad \text { and } \Gamma_{k}^{\delta}=B_{r_{k}}^{n} \times(0, \delta) \quad \text { with } r_{k}=\frac{1}{2}+2^{-k},
$$

and consider

$$
V_{k}=\left|E^{c} \cap\left(\Gamma_{k}^{\delta} \backslash \Gamma_{k+1}^{\delta}\right)\right|
$$

Then, between $\Gamma_{k}$ and $\Gamma_{k+1}$, there is a cylinder $\Gamma_{r}$, such that

$$
\mathscr{H}^{n}\left(\partial \Gamma_{r} \cap E^{c} \cap\{0<z<\delta\}\right) \leq 2^{k} V_{k} .
$$

If we consider $F=E_{c} \cup \Gamma_{r}^{\delta}$, we get

$$
\mathcal{J}(F)-\mathcal{J}(E) \leq-\left(1-\beta_{\max }\right) P\left(E, \Gamma_{r}^{\delta}\right)+\mathscr{H}^{n}\left(\partial \Gamma_{r} \cap E^{c} \cap\{0<z<\delta\}\right)
$$

Since $E$ is a minimizer, the previous quantity must be positive, that is

$$
\left(1-\beta_{\max }\right) P\left(E, \Gamma_{r}^{\delta}\right) \leq \mathscr{H}^{n}\left(\partial \Gamma_{r} \cap E^{c} \cap\{0<z<\delta\}\right) \leq 2^{k} V_{k}
$$

By the isoperimetric inequality, we get

$$
\left|E^{c} \cap \Gamma_{r}\right| \leq \gamma\left(2 P\left(E, \Gamma_{r}^{\delta}\right)+\mathscr{H}^{n}\left(\partial \Gamma_{r} \cap E^{c} \cap\{0<z<\delta\}\right)\right)^{\frac{n+1}{n}}
$$

and thus

$$
V_{k+1} \leq C\left(2^{k} V_{k}\right)^{\frac{n+1}{n}} .
$$

Therefore, $2^{k} V_{k} \longrightarrow 0$ if $V_{0}$ is small enough (That is if $\delta$ is small enough).

This Lemma, together with the monotonicity formula (Lemma 7) allows us to control the perimeter of $E$ in the neighborhood on the contact line:

Corollary 1. If $\left(x_{o}, 0\right) \in \partial E$, then for every $r$,

$$
P\left(E, B_{r}^{+}\left(x_{o}, 0\right)\right) \geq r^{n}
$$


Proof. Let

$$
\begin{aligned}
& V_{1}=\left|E \cap B_{r}\left(x_{o}\right)\right|, \quad S_{1}=\mathscr{H}^{n}\left(E \cap \partial B_{r}\left(x_{o}\right)\right) \\
& V_{2}=\left|B_{r}\left(x_{o}\right) \backslash E\right| \quad S_{2}=\mathscr{H}^{n}\left(\partial B_{r}\left(x_{o}\right) \backslash E\right) \text {. }
\end{aligned}
$$

By the previous lemma, either $B_{r}^{+}\left(x_{o}, 0\right) \backslash\left\{z<\delta_{o} r\right\} \subset E^{c}$, or there exists $\left(y_{o}, z_{o}\right) \in \partial E \cap B_{r / 2}\left(x_{o}, 0\right)$, with $z_{o} \geq \delta_{o} r / 2$. In the first case, we clearly have

$$
V_{2}(r) \geq c r^{n+1}
$$

and

$$
V_{1}(r) \sim\left|E \cap \Gamma_{r}\left(x_{o}\right)\right| \geq c r^{n+1}
$$

by Lemma 6 . In the second case Lemma 7 gives:

$$
V_{1} \geq\left|E \cap B_{\delta_{o} r} y_{o}, z_{o}\right| \geq c\left(\delta_{o} r\right)^{n+1}
$$

and

$$
V_{2} \geq\left|B_{\delta_{o} r} y_{o}, z_{o} \backslash E\right| \geq c\left(\delta_{o} r\right)^{n+1} .
$$

In either case, we deduce

$$
V_{i}(r) \geq c r^{n+1} \quad i=1,2 .
$$

Moreover, the isoperimetric inequality gives

$$
\begin{aligned}
& V_{1}^{\frac{n}{n+1}} \leq \mu_{n+1}\left(S_{1}+P\left(E, B_{r}\left(y_{o}\right)\right)\right) \\
& V_{2}^{\frac{n}{n+1}} \leq \mu_{n+1}\left(S_{2}+P\left(E, B_{r}\left(y_{o}\right)\right)\right)
\end{aligned}
$$

and

$$
\left(2\left(V_{1}+V_{2}\right)\right)^{\frac{n}{n+1}}=\mu_{n+1} 2\left(S_{1}+S_{2}\right)
$$

It follows that

$$
V_{1}^{\frac{n}{n+1}}+V_{2}^{\frac{n}{n+1}}-\left(V_{1}+V_{2}\right)^{\frac{n}{n+1}} \leq \mu_{n+1} P\left(E, B_{r}^{+}\left(y_{o}\right)\right)
$$

which yields the result thanks to (15).

Proposition 4 will now be a consequence of the following Lemma:

Lemma 9. There exists a constant $C$ such that

$$
P(E,\{0<z<t\}) \leq C V^{\frac{n-1}{n+1}} t
$$

Proof of Proposition 4. Let $\cup_{j} B_{\delta}\left(x_{j}\right)$ be a covering of $\partial\{E \cap\{z=0\}\}$ with finite overlapping. Then by Corollary 1, we have

$$
P\left(E, B_{\delta}\left(x_{j}\right)\right) \geq \delta^{n} .
$$

But thanks to the finite overlapping property, 


$$
\sum P\left(E, B_{\delta}\left(x_{j}\right)\right) \leq C P(E,\{0<z<\delta\}) \leq C V^{\frac{n-1}{n+1}} \delta,
$$

and therefore the number of balls is less than $C V^{\frac{n-1}{n+1}} \delta^{1-n}$, hence the result. $\square$

Proof of Lemma 9. Let $F$ the set obtained by cutting $E$ at level $t$ :

$$
F=\left\{(x, z) \in \mathbb{R}^{n+1} ;(x, z+t) \in E\right\} \cap\{z>0\} .
$$

Then

$$
\begin{aligned}
|F| & =|E|-\{E \cap\{0<z<t\}\} \\
& \geq|E|-\omega_{n} R^{n} t
\end{aligned}
$$

and thanks to (14) we have

$$
\mathcal{J}(E) \leq \mathcal{J}(F)+C t
$$

Moreover

$$
\mathcal{J}(E)-\mathcal{J}(F)=P(E,\{0<z<t\})-\int \beta(x / \varepsilon)\left[\varphi_{E}(x, 0)-\varphi_{E}(x, t)\right] d x,
$$

but if $x$ belongs to the symmetric difference of $E \cap\{z=0\}$ and $E \cap\{z=t\}$, then, going from the slice $\{z=0\}$ to the slice $\{z=t\}$, we must cross $\partial E$, and therefore

$$
\int\left|\varphi_{E}(x, 0)-\varphi_{E}(x, t)\right| d x \leq P(E,\{0<z<t\}) .
$$

we deduce

$$
\min \left(1,1-\beta_{\max }\right) P(E,\{0<z<t\}) \leq C V^{\frac{n-1}{n+1}} t
$$

which completes the proof.

\subsection{Sphere-like minimizers}

We can now prove the following result:

Proposition 5. There exists a spherical cap $B_{\rho_{o}}^{+}$such that

$$
\left|E \Delta B_{\rho_{o}}^{+}\right| \leq C\left(V^{\frac{n-1}{n+1}} \varepsilon\right)^{\alpha} .
$$

Moreover, $\left|B_{\rho_{o}}^{+}\right|=V$ and the cosine of the contact angle is $\langle\beta\rangle$.

Proof. We observe that

$$
\int|\langle\beta\rangle-\beta(x / \varepsilon)| \varphi_{E}(x, 0) d x \leq \sum \int_{C_{i}}\left(\beta_{\max }-\beta_{\min }\right) d x
$$


where we sum on all the cells $C_{i}$ of $\varepsilon \mathbb{Z}^{n}$ that intersect the contact line. Thanks to the results of the previous section, the number of such cells cannot exceed $V^{\frac{n-1}{n+1}} \varepsilon^{1-n}$. Since the area of each cell is $\varepsilon^{n}$, we deduce:

$$
-\int\langle\beta\rangle \varphi_{E}(x, 0) d x \leq-\int \beta(x / \varepsilon) \varphi_{E}(x, 0) d x+C V^{\frac{n-1}{n+1}} \varepsilon .
$$

Thus, if we introduce the energy functionnal in which the adhesion coefficient $\beta(x / \varepsilon)$ is replaced by its average $\langle\beta\rangle$ :

$$
\mathcal{J}_{o}(F)=\sigma \iint_{z>0}\left|D \varphi_{F}\right|-\int_{z=0}\langle\beta\rangle \varphi_{F}(x, 0) d x
$$

we have

$$
\mathcal{J}_{o}(E) \leq \mathcal{J}(E)+C V^{\frac{n-1}{n+1}} \varepsilon .
$$

Moreover, if $E_{o}$ denotes the minimizer of $\mathcal{J}_{o}$, we also have

$$
\mathcal{J}\left(E_{o}\right) \leq \mathcal{J}_{o}\left(E_{o}\right)+C V^{\frac{n-1}{n+1}} \varepsilon,
$$

hence (using the fact that $\mathcal{J}(E) \leq \mathcal{J}\left(E_{o}\right)$ )

$$
\mathcal{J}_{o}(E) \leq \mathcal{J}_{o}\left(E_{o}\right)+C V^{\frac{n-1}{n+1}} \varepsilon,
$$

and so $E$ satisfies (7) with $\delta=C V^{\frac{n-1}{n+1}} \varepsilon$, and Theorem 1 gives the proposition.

\subsection{Proof of Theorem 2-(iii)}

We conclude this section by proving that this implies Theorem 2-(iii): It is a consequence of the following nondegeneracy result:

Lemma 10. Let $0<\eta<1 / 2$, then

(i) If there exists $(x, z) \in E \backslash B_{(1+\eta) \rho}^{+}$then

$$
\left|E \backslash B_{\rho}^{+}\right| \geq C(\eta \rho)^{n+1} .
$$

(ii) If there exists $(x, z) \in B_{(1-\eta) \rho}^{+} \backslash E$ then

$$
\left|B_{\rho}^{+} \backslash E\right| \geq C(\eta \rho)^{n+1} .
$$

Proof of Theorem 2-(iii). Let $0<\eta<1 / 2$. Theorem 1 yields that if $\delta=$ $C V^{\frac{n-1}{n+1}} \varepsilon \leq C(\rho \eta)^{(n+1) / \alpha}$ then

$$
\left|E \backslash B_{\rho}^{+}\right|<C(\eta \rho)^{n+1} \quad \text { and }\left|B_{\rho}^{+} \backslash E\right|<C(\eta \rho)^{n+1}
$$

Thus, for $\varepsilon \leq C(V) \eta^{(n+1) / \alpha}$, Lemma 10 implies 


$$
E \backslash B_{(1+\eta) \rho}^{+}=\emptyset, \quad \text { and } B_{(1-\eta) \rho}^{+} \backslash E=\emptyset,
$$

which gives the last part of Theorem 2 .

Proof of Lemma 10. We only give the detailed proof of (i). We have to distinguish the case $z \geq \eta \rho$ and $z \leq \eta \rho$. When $z \geq \eta \rho$, the monotonicity formula Lemma 7 yields

$$
\left|B_{\eta \rho}(x, z) \cap E\right| \geq C(\eta \rho)^{n+1},
$$

and this $B_{\eta \rho}(x, z) \cap B_{\rho}^{+}=\emptyset$, the result follows. When $z \geq \eta \rho$, then the monotonicity formula Lemma 6 gives

$$
\left|\Gamma_{\eta \rho / 2}(x) \cap E\right| \geq C(\eta \rho)^{n+1}
$$

A simple geometric argument shows that if $\eta<1 / 2$, then

$$
\Gamma_{\eta \rho / 2}(x) \cap B_{\rho}^{+}=\emptyset .
$$

The Lemma follows

\section{Stability}

We now turn to the proof of Theorem 1. Throughout this section, we assume that $E$ is such that (6) and (7) hold.

\subsection{Schwartz symmetrisation}

The first step is to prove that $E$ has almost axial symmetry. More precisely, we prove:

Proposition 6. If $E$ is such that (6) and (7) hold, then there exists a universal constant $C$ and $\alpha>0$ such that

$$
\left|E \Delta E^{s}\right| \leq C \delta^{\alpha} .
$$

Moreover, $E^{s}$ also satisfies (7).

For the sake of simplicity, we restrict ourself to the 3 -dimensional case $(n=2)$. We recall that $E^{s}$ denotes the Schwartz symmetrization of $E$ :

$$
E^{s}=\left\{(x, z) ;|x| \leq \rho_{E}(z)\right\}
$$

with

$$
\begin{aligned}
& \rho_{E}(z)=\left(\pi^{-1} A_{E}(z)\right)^{\frac{1}{2}} \\
& A_{E}(z)=\int \varphi_{E}(x, z) d x=\mathscr{H}^{n}\left(E_{z}\right),
\end{aligned}
$$


with the notation

$$
F_{t}=F \cap\{z=t\} .
$$

We recall that $E^{s}$ is a Cacciopolli set satisfying $\left|E^{s}\right|=|E|$ (the schwartz symmetrisation preserves the volume) and so (7) yields

$$
\mathcal{J}_{o}\left(E^{s}\right) \leq \mathcal{J}_{o}(E) \leq \mathcal{J}_{o}\left(E^{s}\right)+\delta .
$$

Since

$$
\beta_{o} \int \varphi_{E}(x, 0) d x=\beta_{o} \int \varphi_{E^{s}}(x, 0) d x
$$

we deduce

$$
P\left(E^{s}, \Omega\right) \leq P(E, \Omega) \leq P\left(E^{s}, \Omega\right)+\delta,
$$

To deduce something on the symmetry of the drop, we establish the following proposition:

Proposition 7. Let $F$ be a subset in $\Omega$, such that $F \subset \Gamma_{R, T}$, and let $F^{*}$ be the set obtained by replacing each horizontal slices of $F$ by a disk with same area and same center of gravity:

$$
F^{*}=\cup_{\{z ; \rho(z) \neq 0\}} D\left(a_{F}(z), \rho_{F}(z)\right),
$$

with

$$
a_{F}(z)=\frac{1}{A_{F}(z)} \int x \varphi_{F}(x, z) d x
$$

Then there exists a constant $C(R, T, P(F))$ such that

$$
F \Delta F^{*} \leq C\left(P(F)-P\left(F^{s}\right)\right)^{1 / 3} .
$$

The proof relies on the following Bonnesen type inequality: If we define the Fraenkel asymmetry of each horizontal slices $F_{z}$ of $F$ by

$$
\lambda_{F}(z)=\frac{\mathcal{H}^{n}\left(F_{z} \Delta D(a(z), \rho(z))\right)}{\mathcal{H}^{n}\left(F_{z}\right)},
$$

then there exists a constant $\mu_{1}$ such that

$$
p(z) \geq 2 \pi \rho(z)\left(1+\mu_{1} \lambda(z)^{2}\right) .
$$

So we need to estimate the isoperimetric default $p(z)-2 \pi \rho(z)$, which will be done using the following lemma, the proof of which is postponed to the appendix:

Lemma 11. Let $F$ be a set in $\Omega$, let $p(z)$ denotes the $(n-1)$-perimeter of the slice $F_{z}$. Let $\rho(z)$ be defined by (18), and let $F^{s}$ be the Schwartz symmetrization of $F$. Then 


$$
P(F) \geq \int \sqrt{p^{2}+\left(2 \pi \rho \rho^{\prime}\right)^{2}} d z
$$

and

$$
P\left(F^{s}\right)=\int \sqrt{(2 \pi \rho)^{2}+\left(2 \pi \rho \rho^{\prime}\right)^{2}} d z
$$

Proof of Proposition 7. Lemma 11, implies

$$
P(F)-P\left(F^{s}\right) \geq \int(p-2 \pi \rho) \frac{2 \pi \rho}{\sqrt{p^{2}+\left(2 \pi \rho \rho^{\prime}\right)^{2}}} d z .
$$

For any borel set $A_{o}$ in $\mathbb{R}_{+}$, we have

$$
\begin{aligned}
\int_{A_{o}} \lambda(z) \mathcal{H}^{n}\left(F_{z}\right) d z & =\int_{A_{o}} \lambda(z) \pi \rho(z)^{2} d z \\
& \leq C\left(\int_{A_{o}} \lambda(z)^{2} \rho^{2} d z\right)^{1 / 2}\left(\int_{A_{o}} \rho(z)^{2} d z\right)^{1 / 2} \\
& \leq C R T^{1 / 2}\left(\int_{A_{o}}(p-2 \pi \rho) \rho d z\right)^{1 / 2}
\end{aligned}
$$

So if $A_{o}$ is the set

$$
A_{o}=\left\{z ; \sqrt{p^{2}+\left(2 \pi \rho \rho^{\prime}\right)^{2}} \geq\left(P(F)-P\left(F^{s}\right)\right)^{-1 / 3}\right\},
$$

we have $\left|A_{o}\right| \leq P(F)\left(P(F)-P\left(F^{s}\right)\right)^{1 / 3}$, and

$$
\begin{aligned}
\int_{\mathbb{R} \backslash A_{o}} \mathcal{H}^{n}\left(F_{z} \Delta D(a(z), \rho(z))\right) d z & =\int_{\mathbb{R} \backslash A_{o}} \lambda \mathcal{H}^{n}\left(F_{z}\right) d z \\
& \leq C R T^{1 / 2}\left(\frac{P(F)-P\left(F^{s}\right)}{\left(P(F)-P\left(F^{s}\right)\right)^{1 / 3}}\right)^{1 / 2} \\
& \leq C R T^{1 / 2}\left(P(F)-P\left(F^{s}\right)\right)^{1 / 3} .
\end{aligned}
$$

It follows that

$$
\left|F \Delta F^{*}\right| \leq C\left(R^{2} P(F)+R T^{1 / 2}\right)\left(P(F)-P\left(F^{s}\right)\right)^{1 / 3}
$$

which gives Proposition 7.

Proof of Proposition 6. If we tried to apply Proposition 7 directly to $E$, we would still have to determine how $E^{s}$ differs from $E^{*}$. This means that we need to control the variations of the center of gravity $a(z)$, which appears to be a delicate task. Instead, we make use of a different approach:

Let $H$ be an hyperplane in $\mathbb{R}^{n+1}$, perpendicular to $\{z=0\}$, and let $H^{+}$ and $H^{-}$be the two half space defined by $H$. By sliding $H$ in the normal direction, we can positioned $H$ in such a way that it cuts the set $E$ into two sets $E^{+}=E \cap H^{+}$and $E^{-}=E \cap H^{-}$with same volume $V / 2$. 
If we denote $E_{1}$ the set formed by adjoining to $E^{+}$its reflexion with respect to $H$, and $E_{2}$ the set form by adjoining to $E^{-}$its reflexion with respect to $H$, we obtain two set $E_{1}$ and $E_{2}$ such that

$$
\left|E_{1}\right|=\left|E_{2}\right|=V, \quad P\left(E_{1}, \Omega\right)+P\left(E_{2}, \Omega\right)=2 P(E, \Omega) .
$$

Repeating the same operation with $E_{1}$ and $E_{2}$, with respect to an hyperplane $H^{\prime}$ perpendicular to $H$ and $\{z=0\}$, we obtained four sets satisfying:

$$
\begin{aligned}
& \left|E_{1}\right|=\left|E_{2}\right|=\left|E_{3}\right|=\left|E_{4}\right|=V \\
& P\left(E_{1}, \Omega\right)+P\left(E_{2}, \Omega\right)+P\left(E_{3}, \Omega\right)+P\left(E_{4}, \Omega\right)=4 P(E, \Omega) .
\end{aligned}
$$

Moreover, each of those set is symmetric with respect to the axis $H \cap H^{\prime}$, which we assume to be given by $x=0$, and therefore

$$
E_{i}^{*}=E_{i}^{s}=\cup_{\left\{z ; \rho_{i}(z) \neq 0\right\}} D\left(0, \rho_{i}(z)\right)
$$

Now, if we denote

$$
S(E)=\int_{\{z=0\}} \varphi_{E}(x, 0) d x,
$$

we have

$$
S\left(E_{1}\right)+S\left(E_{2}\right)+S\left(E_{3}\right)+S\left(E_{4}\right)=4 S(E),
$$

and using (17), we deduce:

$$
\mathcal{J}_{o}\left(E_{1}\right)+\mathcal{J}_{o}\left(E_{2}\right)+\mathcal{J}_{o}\left(E_{3}\right)+\mathcal{J}_{o}\left(E_{4}\right)=4 \mathcal{J}_{o}(E) \leq 4 \mathcal{J}_{o}\left(E_{o}\right)+\delta,
$$

for any $E_{o} \in \mathscr{E}(V)$. If we choose $E_{o}$ to be a minimizer for $\mathcal{J}_{o}$, we also have $\mathcal{J}_{o}\left(E_{o}\right) \leq \mathcal{J}_{o}\left(E_{i}\right)$ for each $i=1 \cdots 4$, and thus

$$
\mathcal{J}_{o}\left(E_{i}\right) \leq \mathcal{J}_{o}\left(E_{o}\right)+\delta, \quad i=1 \cdots 4 .
$$

But $P\left(E_{i}^{s}\right) \leq P\left(E_{i}\right)$ and $S\left(E_{i}^{s}\right)=S\left(E_{i}\right)$; therefore

$$
P\left(E_{i}\right)-P\left(E_{i}^{s}\right) \leq \delta, \quad i=1 \cdots 4 .
$$

Proposition 7, together with (23) implies

$$
\left|E_{i} \Delta E_{i}^{s}\right| \leq C \delta^{1 / 3} \quad i=1 \cdots 4 .
$$

In order to conclude, we now reconstruct the set

$\widetilde{E}^{s}=\left(E_{1}^{s} \cap H^{+} \cap H^{\prime+}\right) \cup\left(E_{2}^{s} \cap H^{+} \cap H^{\prime-}\right) \cup\left(E_{3}^{s} \cap H^{-} \cap H^{\prime+}\right) \cup\left(E_{4}^{s} \cap H^{-} \cap H^{\prime-}\right)$,

We clearly have $\left|E \Delta \widetilde{E}^{s}\right| \leq C \delta^{1 / 3}$, so we only have to check that 


$$
\left|E^{s} \Delta \widetilde{E}^{s}\right| \leq C \delta^{1 / 3} .
$$

To that purpose, we need to show that for a given $z$, the slices $E_{i z}^{s}$ have almost the same radii for $i=1 \cdots 4$. This is a consequence of the strict convexity of the square function: We note that the sum of the area of the slices $E_{i z}$ is equal to four times the area of $E_{z}$, and the same is true for the perimeter. In other words:

$$
\begin{aligned}
& \sum p_{i}(z)=4 p(z) \\
& \sum \rho_{i}^{2}=4 \rho^{2}
\end{aligned}
$$

where $p_{i}$ and $\rho_{i}$ denote respectively the perimeter of $E_{i z}$ and the radius of $E_{i z}^{s}$. Thus, if we denote by $\mu_{i}=p_{i}-2 \pi \rho_{i}$ the defect in the isoperimetric inequality for the slice $E_{i z}$, we have:

$$
2 \pi \rho \leq p=\frac{1}{4} \sum p_{i} \leq \frac{1}{4} \sum\left(2 \pi \rho_{i}+\mu_{i}\right) .
$$

It follows that

$$
\frac{1}{4} \sum \rho_{i}^{2} \leq\left(\frac{1}{4} \sum \rho_{i}+\mu_{i}\right)^{2}
$$

by strict convexity of the square function, we deduce:

$$
\sum\left|\rho_{i}-\rho_{j}\right|^{2} \leq \sum \mu_{i}^{2}+\sum \mu_{i} \rho_{j}, \quad i=1 \cdots 4
$$

Proceeding as in the proof of Proposition 7, we show that

$$
\int_{A_{o}} \mu_{i}^{2} d z \leq \int_{A_{o}} \mu_{i} p_{i} d z \leq C\left(P\left(E_{i}\right)-P\left(E_{i}^{s}\right)\right)^{2 / 3}
$$

with $A_{o}=\left\{z ; \sqrt{p^{2}+\left(2 \pi \rho \rho^{\prime}\right)^{2}} \geq\left(P\left(E_{i}\right)-P\left(E_{i}^{s}\right)\right)^{-1 / 3}\right\}$. We deduce that

$$
\int_{\mathbb{R} \backslash A_{o}}\left|\rho_{i}-\rho_{j}\right|^{2} d z \leq C \delta^{2 / 3}
$$

which implies that

$$
\int_{\mathbb{R} \backslash A_{o}}\left|\rho_{i}^{2}-\rho^{2}\right| d z \leq C \delta^{1 / 3} .
$$

Since $\left|A_{o}\right| \leq C \delta^{1 / 3}$, the result follows.

\subsection{Sphere-like minimizer}

Proposition 6 shows that $E$ has almost axial symmetry. We now complete the proof of Theorem 1 by showing that a symmetric set satisfying (7) cannot differ too much from a spherical cap: 
Proposition 8. There exists a ball $B_{\rho_{o}}\left(x_{o}, z_{o}\right)$ in $\mathbb{R}^{n+1}$ such that

$$
\left|E \Delta\left(B_{\rho_{o}}\left(x_{o}, z_{o}\right) \cap \Omega\right)\right| \leq C \delta^{\alpha} .
$$

Moreover, $B_{\rho_{o}}\left(x_{o}, z_{o}\right) \cap \Omega$ is the minimizer associated to the averaged functional $\mathcal{J}_{o}$ and is fully determined by the conditions

$$
\left|B_{\rho_{o}} \cap \Omega\right|=V, \quad \cos \gamma=\langle\beta\rangle .
$$

Proof. For any given ball $B_{\rho}(x, z)$, we denote

$$
B_{\rho}^{+}=B_{\rho}(x, z) \cap \Omega \quad \text { and } \quad B_{\rho}^{-}=B_{\rho}(x, z) \backslash \Omega .
$$

In view of the result of the previous section, we only have to show that

$$
\left|E^{s} \Delta B_{\rho_{o}}^{+}\right| \leq C \delta^{\alpha}
$$

where $E^{s}$ denotes the Schwartz symmetrisation of $E$. Again, after rescaling, we assume that $V=1$.

Let $\Sigma$ denotes the wetting surface

$$
\Sigma=E^{s} \cap\{z=0\} .
$$

Note that $\Sigma$ is a disk, so there exists a ball $B_{\rho}\left(x_{o}, z_{o}\right)$ in $\mathbb{R}^{n}$ satisfying

$$
B \cap\{z=0\}=\Sigma, \quad|B \cap\{z>0\}|=V .
$$

To establish inequality (25), we compare the perimeter of $B$ and that of the set obtained by adjoining $B_{\rho}^{-}=B_{\rho}\left(x_{o}, z_{o}\right) \cap\{z<0\}$ to $E^{s}$ :

$$
G=E^{s} \cup B_{\rho}^{-} .
$$

We have

$$
\begin{aligned}
\int_{\mathbb{R}^{n+1}}\left|D \varphi_{G}\right| & =\mathcal{J}_{o}\left(E^{s}\right)+\mathscr{H}^{n}(\Sigma) \beta_{o}+\int_{\{z<0\}}\left|D \varphi_{B_{\rho}^{-}}\right| \\
\int_{\mathbb{R}^{n+1}}\left|D \varphi_{B_{\rho}}\right| & =\mathcal{J}_{o}\left(B_{\rho}^{+}\right)+\mathscr{H}^{n}(\Sigma) \beta_{o}+\int_{\{z<0\}}\left|D \varphi_{B_{\rho}^{-}}\right|
\end{aligned}
$$

Moreover, (7) implies

$$
\mathcal{J}_{o}(E) \leq \mathcal{J}_{o}\left(B_{\rho}^{+}\right)+\delta
$$

and since $\mathcal{J}_{o}\left(E^{s}\right) \leq \mathcal{J}_{o}(E)+\delta$, we deduce

$$
\int_{\mathbb{R}^{n+1}}\left|D \varphi_{G}\right| \leq \int_{\mathbb{R}^{n+1}}\left|D \varphi_{B_{\rho}}\right|+\delta
$$

or

$$
0 \leq P\left(G, \mathbb{R}^{n+1}\right)-P\left(B_{\rho}, \mathbb{R}^{n+1}\right) \leq C \varepsilon
$$


which measure the quantitative loss in the isoperimetric inequality.

R.R. Hall proved in [Hal92] that if we introduce

$$
\lambda(F)=\sup _{a} \frac{\left|F \cap B_{\rho}(a)\right|}{\left|B_{\rho}(a)\right|}
$$

with $\rho$ such that $\left|B_{\rho}(a)\right|=|F|$, then there exists a constant $c$ such that

$$
P(F) \geq P\left(B_{\rho}\right)\left(1+c \lambda(F)^{4}\right) .
$$

Moreover, the exponent 4 can be replaced by 2 in the case of axially symmetric domains (which is the case of $G$ ). Therefore, we have

$$
\lambda(G) \leq C \sqrt{\frac{P(E)-P\left(B_{\rho}\right)}{P\left(B_{\rho}\right)}}
$$

Finally, since $V=1$, the measure of the wetting surface $\mathscr{H}^{n}(\Sigma)$ is bounded, and so the radius $\rho$ (and therefore $P\left(B_{\rho}\right)$ ) is bounded. It follows that

$$
\lambda(G) \leq C \delta^{1 / 2}
$$

which gives (25).

To complete the proof of Proposition 8, it remains to see that

$$
\left|B_{\rho}^{+} \Delta B_{\rho_{o}}^{+}\right| \leq C \delta
$$

where $B_{\rho_{o}}^{+}$is a spherical cap with volume $V$ such that the cosine of the contact angle is $\beta_{o}$. First, we notice that $B_{\rho}^{+}$satisfies (7), and in particular

$$
\mathcal{J}_{o}\left(B_{\rho}^{+}\right) \leq \mathcal{J}_{o}\left(B_{\rho_{o}}^{+}\right)+C \delta .
$$

So the result will follow from a simple computation: If $B_{r}^{+}$is a spherical cap with radius $r$ and contact angle $\gamma$ (see Figure 1), the volume conditions yields

$$
r=\left(\frac{3 V}{\pi(2+\cos \gamma)(\cos \gamma-1)^{2}}\right)^{1 / 3}
$$

which implies

$$
\mathcal{J}_{o}\left(B_{r}^{+}\right)=\mathscr{J}(\cos \gamma)=\frac{\pi^{1 / 3}(3 V)^{2 / 3}}{(\cos \gamma+2)^{2 / 3}(\cos \gamma-1)^{1 / 3}}\left(\beta_{o}(1+\cos \gamma)-2\right) .
$$

We deduce:

$$
\mathscr{J}^{\prime}(\cos \gamma)=\frac{2 \pi^{1 / 3}(3 V)^{2 / 3}}{(\cos \gamma-1)^{4 / 3}(\cos \gamma+2)^{5 / 3}}\left(\cos \gamma-\beta_{o}\right)
$$




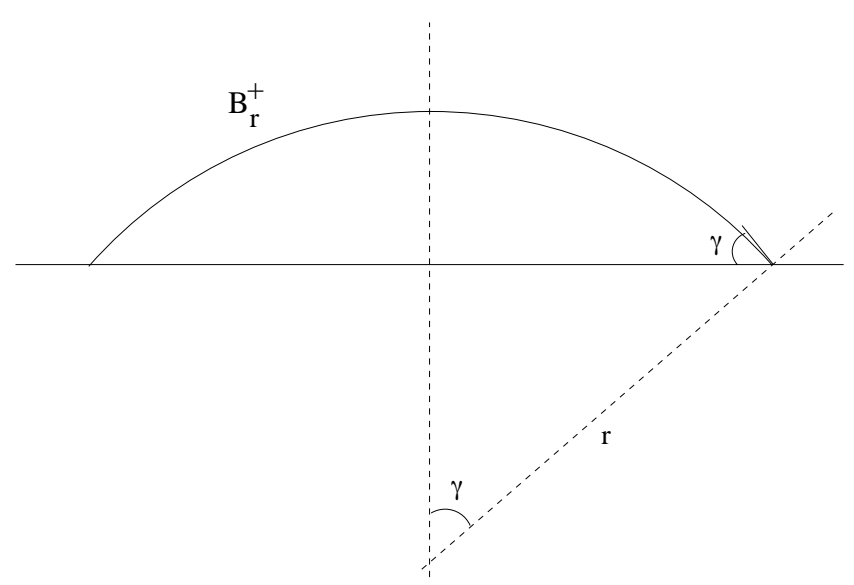

Fig. 1. $B_{r}^{+}$

In particular, the only minimum is reached when $\cos \gamma=\beta_{o}$, and since

$$
\mathscr{J}^{\prime \prime}\left(\beta_{o}\right)=\frac{2 \pi^{1 / 3}(3 V)^{2 / 3}}{\left(\beta_{o}-1\right)^{4 / 3}\left(\beta_{o}+2\right)^{5 / 3}}>0
$$

we deduce that for every $\beta_{o} \in(-1,1)$, there exists a constant $C$ such that

$$
\mathcal{J}_{o}\left(B_{r}^{+}\right)-\mathcal{J}_{o}\left(B_{\rho_{o}}^{+}\right) \geq C\left|\cos \gamma-\beta_{o}\right|^{2}
$$

and so (27) gives that the contact angle $\gamma$ of $B_{\rho}^{+}$satisfies

$$
\left|\cos \gamma-\beta_{o}\right|^{2} \leq C \delta .
$$

Next, we recall that $\beta_{o}<1$, and so either $\cos \gamma \leq\left(1+\beta_{o}\right) / 2$, in which case (28) yields

$$
\left|\rho-\rho_{o}\right| \leq C\left(\beta_{o}\right)\left|\cos \gamma-\beta_{o}\right|,
$$

or $\cos \gamma \geq\left(1+\beta_{o}\right) / 2$ and in particular $\left|\cos \gamma-\beta_{o}\right| \geq\left(1-\beta_{o}\right) / 2$ which yields

$$
\delta \geq C\left(\beta_{o}\right) .
$$

In either case, using (27), we deduce the existence of constants $C$ and $\alpha$ such that

$$
\left|B_{\rho}^{+} \Delta B_{\rho_{o}}^{+}\right| \leq C \delta^{\alpha},
$$

so the proof of Proposition 8 is complete. 


\section{A Proof of Lemma 11.}

Let $n(x, z)$ be an extension of the unit outward normal vector to $\partial F_{z}$ in $\mathbb{R}^{n}$, and take

$$
\Phi(x, z)=(u(z) n(x, z), v(z))
$$

a test function, with $u(z)$ and $v(z)$ satisfying

$$
|u(z)|^{2}+|v(z)|^{2} \leq 1 \quad \text { for all } z \in \mathbb{R} .
$$

Then

$$
\begin{aligned}
\int \varphi_{F} \operatorname{div}_{x, z} \Phi d x d z & =\iint \varphi_{F} \operatorname{div}_{x}(n(x)) d x u(z) d z+\iint \varphi_{F} d x \partial_{z} v(z) \\
& =-\int p(z) u(z)-\int A^{\prime}(z) v(z) d z
\end{aligned}
$$

Taking

$$
u(z)=\frac{p(z)}{\sqrt{p^{2}+A^{\prime 2}}} \quad \text { and } \quad v(z)=\frac{A^{\prime}(z)}{\sqrt{p^{2}+A^{\prime 2}}}
$$

we check that $|u(z)|^{2}+|v(z)|^{2} \leq 1$ and therefore

$$
\int\left|D \varphi_{F}\right| \geq \int \sqrt{p^{2}+\left(2 \pi \rho \rho^{\prime}\right)^{2}} d z
$$

We now prove that there is in fact equality in the case of the Schwartz symmetrisation $F^{s}$ : Let $\varphi(x, z)$ be a test function, then:

$$
\int \varphi_{F} \frac{\partial \varphi}{\partial z} d x d z=\iint_{D(0, \rho)} \frac{\partial \varphi}{\partial z} d x d z
$$

and if we denote $\nu(\theta)=(\cos \theta, \sin \theta)$ the unit normal vector, we have

$$
\begin{aligned}
\frac{\partial}{\partial z} \int_{D(0, \rho)} \varphi(x, z) d x= & \frac{\partial}{\partial z} \int_{0}^{\rho} \int_{0}^{2 \pi} \varphi(r \nu, z) r d \theta d r \\
= & \iint_{D(0, \rho)} \frac{\partial \varphi}{\partial z} d x \\
& +\int_{0}^{2 \pi} \rho^{\prime} \varphi(\rho \nu, z) \rho d \theta
\end{aligned}
$$

Therefore

$$
\int \varphi_{F^{s}} \frac{\partial \varphi}{\partial z} d x d z=-\iint_{0}^{2 \pi} \rho\left(\rho^{\prime}\right) \varphi(\rho \nu, z) d \theta d z
$$

Next, for all $\phi \in\left(\mathcal{C}\left(\mathbb{R}^{n+1}\right)\right)^{2}$ we have 


$$
\begin{aligned}
\int \varphi_{E^{s}} \operatorname{div}_{x} \phi d x d z & =\iint_{D(0, \rho)} \operatorname{div}_{x} \phi d x d z \\
& =-\iint_{0}^{2 \pi} \phi(\rho \nu, z) \cdot \nu \rho d \theta d z
\end{aligned}
$$

Thus, if $\Phi=(\phi, \varphi)$ satisfies $|\Phi| \leq 1$, we have

$$
\begin{aligned}
\int \varphi_{F} \operatorname{div}_{x, z} \Phi d x d z= & -\iint_{0}^{2 \pi} \rho\left(\rho^{\prime}\right) \varphi(\rho \nu, z) d \theta d z \\
& -\iint_{0}^{2 \pi} \phi(\rho \nu, z) \cdot \nu \rho d \theta d z \\
= & -\iint_{0}^{2 \pi}\left(\begin{array}{c}
\rho \\
\rho \rho^{\prime}
\end{array}\right) \cdot\left(\begin{array}{c}
\phi \cdot \nu \\
\varphi
\end{array}\right)(\rho \nu, z) d \theta d z
\end{aligned}
$$

and so

$$
\left|\int \varphi_{F_{s}} \operatorname{div}_{x, z} \Phi d x d z\right| \leq \int 2 \pi \sqrt{\rho^{2}+\left(\rho \rho^{\prime}\right)^{2}} d z .
$$

\section{References}

[Bar83] Elisabetta Barozzi. The Plateau problem in unbounded domains. Rend. Sem. Mat. Univ. Padova, 70:89-98, 1983.

[BG84] E. Barozzi and E. H. A. Gonzalez. Least area problems with a volume constraint. Astérisque, 118:33-53, 1984. Variational methods for equilibrium problems of fluids (Trento, 1983).

[CM] L. A. Caffarelli and A. Mellet. Capillart drops on an inhomogeneous surface: Contact angle hysteresis and sticking drop. Submitted.

[Fin86] Robert Finn. Equilibrium capillary surfaces, volume 284 of Grundlehren der Mathematischen Wissenschaften [Fundamental Principles of Mathematical Sciences]. Springer-Verlag, New York, 1986.

[Giu84] Enrico Giusti. Minimal surfaces and functions of bounded variation, volume 80 of Monographs in Mathematics. Birkhäuser Verlag, Basel, 1984.

[GMT83] E. Gonzalez, U. Massari, and I. Tamanini. On the regularity of boundaries of sets minimizing perimeter with a volume constraint. Indiana Univ. Math. J., 32(1):25-37, 1983.

[Gon76] Eduardo H. A. Gonzalez. Sul problema della goccia appoggiata. Rend. Sem. Mat. Univ. Padova, 55:289-302, 1976.

[Hal92] R. R. Hall. A quantitative isoperimetric inequality in $n$-dimensional space. J. Reine Angew. Math., 428:161-176, 1992.

[HM77] C. Huh and S. G. Mason. Effects of surface roughness on wetting (theoretical). J. Colloid Interface Sci., 60:11-38, 1977.

[JdG84] J. F. Joanny and P. G. de Gennes. A model for contact angle hysteresis. J. Chem. Phys., 81, 1984.

[LJ92] L. Leger and J. F. Joanny. Liquid spreading. Rep. Prog. Phys., pages 431-486, 1992. 